\title{
Research on the Teaching Methods of Database Principle based on E-learning
}

\author{
Fang Dong ${ }^{1}$, Wenfeng Wang ${ }^{2}$, Yangbo Jiang ${ }^{3}$ \\ ${ }^{1)}$ Department of Scientific Research, Nanchang Institute of Technology, Nanchang 330099, China; \\ ${ }^{2)}$ School of Information Engineering, Nanchang Institute of Technology, Nanchang 330099, China \\ ${ }^{3)}$ Network information center, Nanchang Institute of Technology, Nanchang 330099, China \\ dongf@nit.edu.cn, wangwf@nit.edu.cn
}

\begin{abstract}
Autonomy, interactivity, cooperativity, flexibility and etc., all these luxuries contribute to the popularity of online learning. However, great changes have taken place in the learning subjects and learning objects, as well as the learning tools. Learners usually obtained limited networked learning resources by wired-network-accessed computers in the past, while they can get massive diversified resources by mobile phones. In this paper, we firstly present the online learning data of undergraduates from the Nanchang Institute of Technology, and then we analyze the learning behavior. Finally, we propose some strategies. Teaching practice demonstrates that they are effective to promote the quality of teaching.
\end{abstract}

Keywords —Database principle, E-learning, Heuristic teaching, Project teaching

\section{基于 E-Learning 的数据库原理教学方法研究 ${ }^{*}$}

\author{
董芳 ${ }^{1}$ 王文丰 $^{2}$ 江阳波 $^{3}$ \\ 1) 南昌工程学院科学研究处, 江西 南昌 330099 \\ 2) 南昌工程学院信息工程学院, 江西 南昌 330099 \\ 3) 南昌工程学院 网络信息中心, 江西 南昌 330099
}

摘 要 针对 E-Learning 环境下如何开展数据库原理课程教学问题, 本文分别从课堂教学和课外教学两个方面进行了介绍, 重点阐 述了启发式、讨论式、案例式和项目驱动式教学方式的设计原则, 并给出了具体的应用。教学实践表明, E-Learning 教学方式有效提 高了学生的学习积极性, 激发了学生的学习热情, 具有良好的教学效果。

关键词 数据库原理, E-Learning, 启发式教学, 项目教学法

1. 引言

当今世界是一个信息爆炸的时代, 传统的教学模式已 经不能满足有限时间内讲授大信息量教学内容的要求。现 代信息技术特别是网络技术的飞速发展, 使得互联网不仅 成为资源的海洋, 同时更成为最便捷的交流工具。在这种 情况下, 越来越多的人开始通过网络进行学习。

E-Learning 是指通过因特网或其他信息技术进行学习 与教学活动的一种方式。它充分利用现代信息技术所提供
的、具有全新沟通机制与丰富资源的学习环境, 实现一 种全新的学习方式。自上世纪 90 年代开始, E-Learning 逐渐从企业进入学校。近年来, 国内外许多高校纷纷利用 多媒体、网络等信息化技术手段, 对传统教学方式进行改 革。它们不仅为许多课程建立了相应的网络课堂, 而且还 建立了功能强大的网络教学综合平台, 以便于在所有课程 中开展网络教学。从某种程度上来讲, 借助各类网络平台 开展教学活动, 这已经成为现代教学方式的一种发展趋势。

*江西省教育科学 “十二五” 规划 2012 年度重点课题 “E-Learning 环境下大学生在线学习行为特征研究一以南昌工程学院为例” (编号: 12ZD073), 南昌工程学院校级教学改革研究项目资助。 
数据库原理是我校计算机科学与技术、信息管理与信 息系统以及软件工程三个专业的核心基础课程。它具有信 息量大、知识更新快等特点, 因此非常适合且很有必要开 展 E-Learning 教学。由于 E-Learning 改变了传统教学中教 师的作用和师生之间的关系, 也即从根本上改变了教学结 构和教育本质。因此, 迫切需要研究一种与之相适应的教 学模式。本文将立足我校培养高级应用型人才这一目标, 结合近年来的教学实践, 探讨在 E-Learning 环境下如何开 展数据库原理课程的教学方式研究。

\section{2. 基于 E-Learning 的数据库原理教学方式}

数据库原理是我校计算机科学与技术、信息管理与信 息系统以及软件工程三个专业的核心基础课程。它具有信 息量大、知识更新快等特点, 因此非常适合且很有必要开 展 E-Learning 教学。由于 E-Learning 改变了传统教学中教 师的作用和师生之间的关系, 也即从根本上改变了教学结 构和教育本质。因此, 迫切需要研究一种与之相适应的教 学模式。本文将立足我校培养高级应用型人才这一目标, 结合近年来的教学实践, 探讨在 E-Learning 环境下如何开 展数据库原理课程的教学方式研究。

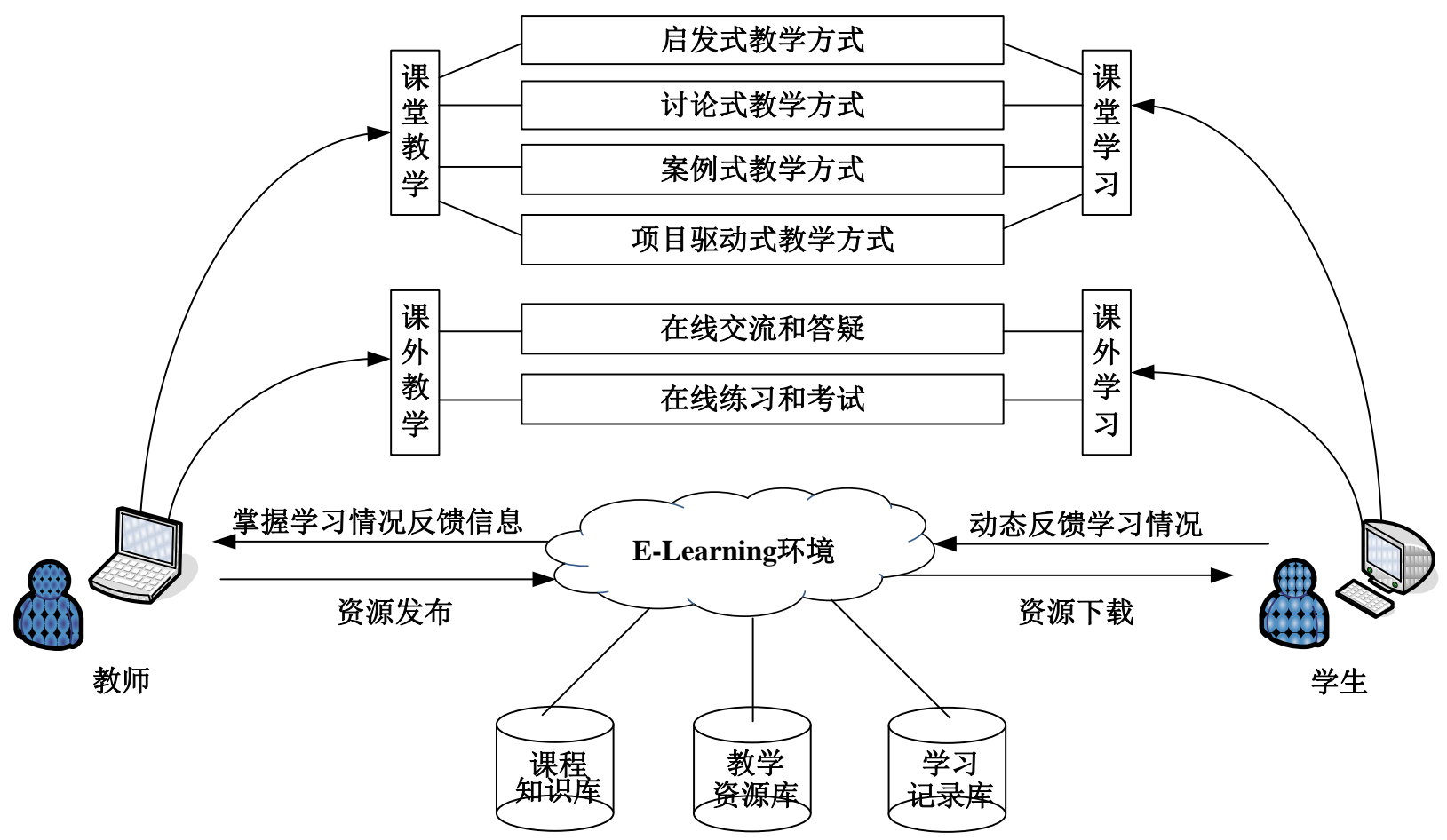

图 1 基于 E-Learning 的数据库原理教学方式

\section{1 课堂教学方式}

\section{1. 启发式课堂教学方案设计}

启发式教学注重培养学生自主学习和创新精神, 对出 现的问题不是直接给出答案, 而是指明一个方向引导学生 通过资料查询、组内讨论等多种方式找出答案。具体方案 为:

(1) 分析数据库原理教学内容, 有针对性地设计一系 列的启发式教学点;

(2) 对启发式教学点进行分类: 可以当堂启发和当堂 解决的, 需要当堂启发但课外解决的。针对不同类型的启 发式教学点，设计每个启发式教学点的启发过程;

(3) 利用清华大学教育技术研究所开发的网络教学综
合平台（以下简称“网教平台”）及时掌握学生学习情况， 了解学生在学习过程中存在的问题;

（4）根据学生学习情况反馈信息, 不断调整和优化启 发过程，并修改完善或重新设计启发式教学点;

（5）重复执行步骤（2）至（4), 直至完成预定教学 目标。

2. 讨论式课堂教学方案设计

讨论式教学是由教师预先设定讨论题目和要求, 由学 生分组讨论的一种互动式教学方式。具体方案如下:

(1) 首先分析和研究数据库原理课程中适合开展讨论 式教学的重难点教学内容, 并据此设计若干个讨论题目, 并制定详细的讨论要求;

（2）将讨论题目在网教平台上进行发布，要求 4-6人 
分为一组, 针对各个讨论题目, 按照相应的讨论要求, 利 用课外时间预先收集资料，并进行较为充分的前期讨论;

(3) 在课堂内进行分组讨论和组间交叉讨论, 教师分 小组点评, 并做最后总结。
表 1 给出了笔者在实际教学过程中针对讨论式教学方 式设计的若干个讨论主题, 所采用的教程为参考文献[4]中 的教材。

表 1 讨论式教学主题设计

\begin{tabular}{|c|l|c|}
\hline 序号 & \multicolumn{1}{|c|}{ 讨论主题 } & 所属章节 \\
\hline 1 & 外连接、左/右外连接、自然连接和等值连接之间的关系 & 第二章 \\
\hline 2 & 关系模式的规范化程度是否越高越好 & 第六章 \\
\hline 3 & XXXX 数据库系统需求分析设计方法 & 第七章 \\
\hline 4 & 建立索引的基本原则 (实例说明) & 第九章 \\
\hline$\ldots$ & & \\
\hline
\end{tabular}

\section{3. 案例式课堂教学方案设计}

案例教学起源于“哈佛大学”的情景案例教学, 它通过 具体的情境促进隐性知识与显性知识的不断转化。设计 1-2 个典型的数据库应用案例, 将其贯彻于数据库原理课程教 学和实践教学的整个过程。具体方案如下:

(1) 精选和设计 1-2 个典型的数据库应用案例, 其中 包括数据库设计和应用程序设计的详细文档, 程序源代码 和可运行演示的执行代码 (教学过程中选择“学生选课信息 管理系统” 作为案例, 同时提供 Java 和 C\#.NET 两种高级语 言编写的完整源程序和文档);

(2) 针对不同的课堂教学内容, 采用应用程序演示、 剖析设计方案和分析程序源码等各种方式设计新的课堂教 学过程;

(3) 针对选定的典型数据库应用案例和数据库原理不 同章节的教学内容, 设计和设置具体的实验内容和要求, 提高实践课程教学效果。

\section{4. 项目驱动式课堂教学方案设计}

按照 4-5 人为标准对学生进行分组, 要求在课程开设 2 周内自主拟定一个项目名称, 设计“XXXX 信息管理系统” 这一应用项目, 将其贯穿于整个数据库原理课程的教学过 程中, 以此激发学生学习的积极性。具体方案为:

(1) 根据教学大纲对数据库原理课程的培养目标和要 求, 从宏观上设计好“XXXX 信息管理系统”项目的任务和 要求, 力争涵盖到所有知识点;

(2) 按照项目的设计实施过程, 制定好相应的授课计 划和教学安排;
(3) 在理论课程教学结束后, 以学生自主设计为主、 教师指导为辅, 进行为期两周的数据库专题实训, 完成项 目设计和实现任务，从而将理论和实践相结合。

需要特别说明的是, 由于数据库原理这门课程一般在 第四学期才进行开设, 大多数学生此时尚不具备 C++、Java 或.NET 等高级语言编程方面的知识, 因此在实际教学中需 要让学生提前自主学习。

\section{2 课外教学方式}

\section{1. 在线交流和答疑}

利用校园网教平台, 开辟《数据库原理》课程的学习 讨论专区。学生能够进行在线交流、学习和讨论, 并及时 将学习情况进行反馈。通过在线答疑、问卷调查等形式, 教师能够及时掌握学生的学习情况, 能够对所提问题进行 答疑, 并据此对教学方式和内容进行动态调整。

\section{2. 在线练习和考试}

开展《数据库原理》试题库和试卷库的研究与设计工 作, 将其在网教平台上进行发布, 让学生能够在平时的学 习过程中通过在线练习对所学知识及时进行巩固, 同时还 可以在学期末通过在线模拟考试进行系统化的复习。

\section{3 教学效果}

目前, 笔者从事《数据库原理》课程教学工作已逾五 年, 其中从 2011 年开始采用 E-Learning 教学方式授课, 累 计达 2000 多人次。 


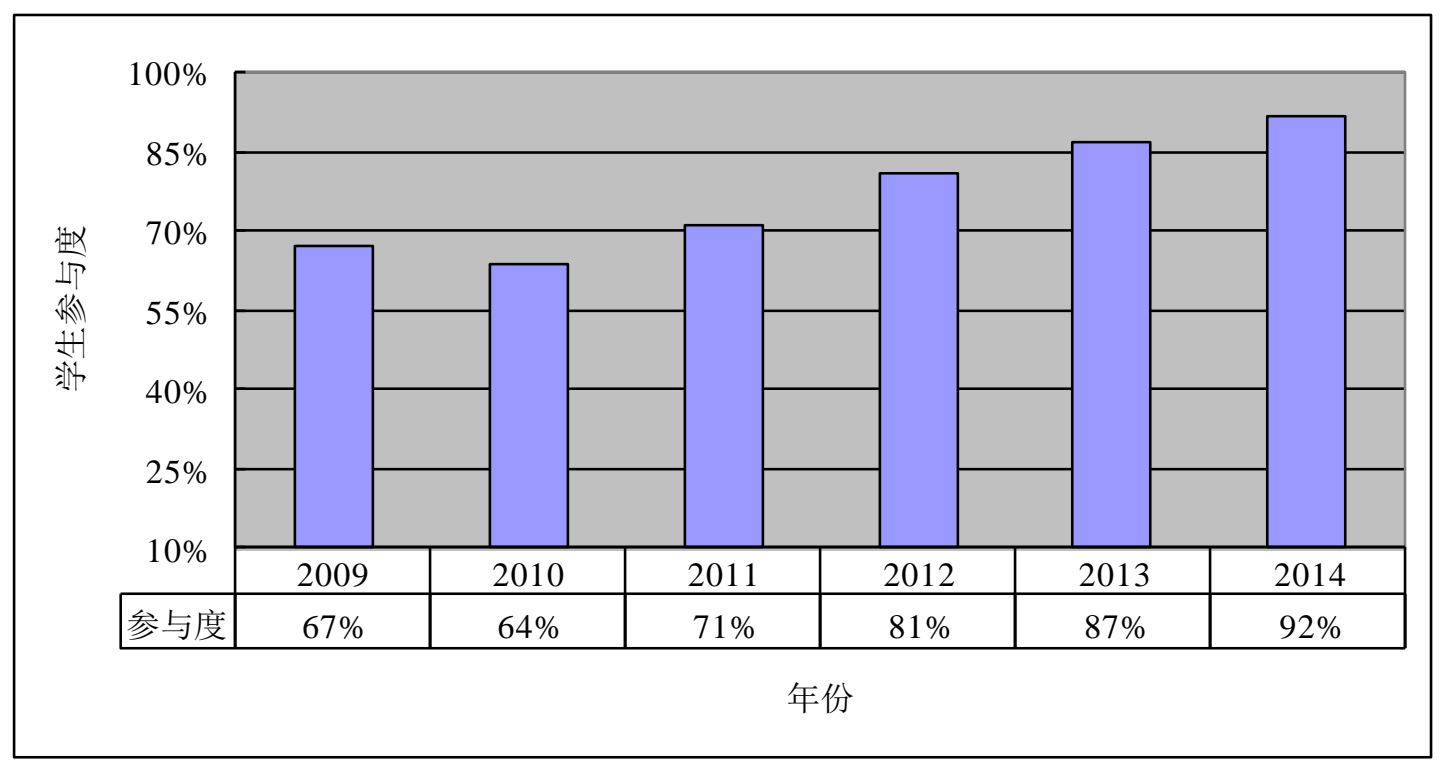

图 2 E-Learning 授课前后学生课堂参与度情况

图 2 给出了 E-Learning 授课前后学生课堂参与度的变 化情况。从教学实践情况来看, 学生课堂参与度和学习积 极性较以往有显著提高, 特别是在网络课堂上表现尤为突 出。此外, 学生的综合能力 (如: 数据库理论与技术、面 向对象编程、软件工程、交流与沟通以及文档撰写等) 也 得到了充分的锻炼和提高, 全面达到甚至超出了预期的教 学目标。

\section{3. 结束语}

数据库原理是一门专业基础课程, 它在整个课程体系 中的地位举足轻重, 特别是在后续的基于数据库系统的软 件开发等实践能力培养环节中有着非常重要的作用。本文 将 E-Learning 先进的教育理念引入到数据库原理教学过程 中, 分别从课堂教学和课外教学两个方面进行了详细阐述。 教学实践表明, E-Learning 教学方式有效提高了学生的学习 积极性, 激发了学生的学习热情, 具有良好的教学效果。 下一步将考虑如何将该教学方式在其它班级和专业进行推 广应用。

\section{参考文献(References)}

[1] Kebin Huang. Feng Wang. The network learning behavior study under E-learning environment. Journal of Shanxi Radio \& TV University, 2008, 2: 18-19

[2] Shu Na, Liu Jing. The Impact of Learner Factor on E-Learning Quality. Proc. of Int'l Conf. on E-Learning, E-Business, Enterprise Information Systems, and E-Government, 2009: 111-114

[3] Peng Wenhui, Li Suzhen, Zhu Wanying. Investigation and Analysis on E-learning Behavior of Spare-time Students. Proc. of Int'l Conf. on Internet Computing and Information Services, 2011: 381-384

[4] Shixuan Sa, Shan Wang. Introduction to Database Systems(Fourth Edition) BeiJing: Higher Education Press, 2006

\section{作者简介}

董芳(1984-), 女, 湖北黄冈人, 硕士, 讲师; 王文丰(1983-), 男, 湖北黄冈人, 博士, 副教授; 江阳波(1978-), 男, 江西丰城人, 工程师

Email: sunbird2002@126.com 